Article

\title{
Evaluation of Preemergence and Postemergence Herbicide Programs on Weed Control and Weed Seed Suppression in Mississippi Peanut (Arachis hypogea)
}

\author{
John W. Seale *, Taghi Bararpour*, Jason A. Bond, Jeffrey Gore and Bobby R. Golden \\ Delta Research and Extension Center, Mississippi State University, Stoneville, MS 38776, USA; \\ jbond@drec.msstate.edu (J.A.B.); jgore@drec.msstate.edu (J.G.); bgolden@drec.msstate.edu (B.R.G.) \\ * Correspondence: jws764@msstate.edu (J.W.S.); mtb436@msstate.edu (T.B.)
}

Received: 29 June 2020; Accepted: 18 July 2020; Published: 22 July 2020

\begin{abstract}
Weed control and reducing weed seed deposition to the soil seedbank is a challenging issues for Mississippi peanut producers. Research was established during 2017 and 2018 at the Delta Research and Extension Center in Stoneville, Mississippi, to evaluate herbicide programs for weed control and reducing weed seed production in Mississippi peanut production. Treatments were combinations of acetochlor, clethodim, flumioxazin, lactofen, paraquat, and S-metolachlor with their respective adjuvants if needed. Treatments were applied PRE, two to three weeks after emergence (EPOST), and/or four to five weeks after emergence (MPOST). All treatments included a PRE application followed by (fb) application of EPOST and/or MPOST application. Flumioxazin PRE fb lactofen plus clethodim MPOST provided greater than or equal to $(\geq) 88 \%$ control of barnyardgrass, hemp sesbania, Palmer amaranth, pitted morningglory, and prickly sida. Additionally, this treatment reduced total weed seed production $88 \%$ compared to the nontreated control. Flumioxazin PRE fb lactofen plus clethodim EPOST fb acetochlor MPOST provided similar weed control and peanut yield as flumioxazin PRE fb lactofen plus clethodim MPOST. This treatment reduced total weed seed production $93 \%$. Treatments containing PRE, EPOST, and MPOST herbicide applications provided the best season-long control of weeds and weed seed suppression in Mississippi peanut.
\end{abstract}

Keywords: soil seedbank; dry weight; seed production; weed control

\section{Introduction}

Weed control is essential for maximizing peanut yield [1,2]. Competitive nature and allelopathic interactions are major characteristics of weeds that influence crop yield [1]. Research has suggested a relationship between crops and weeds resulting in peanut yield loss from weed interference [3-5]. The relationship between crops and weeds can be explained by the critical period for weed control (CPWC) [6]. The CPWC is the period of time during the crop growth cycle in which weeds must be controlled to prevent peanut yield loss $\geq 5 \%[2,6,7]$. Studies have reported the first three to five weeks of the growing season without weed competition will allow maximum crop yields if environmental conditions are favorable [8]. Studies have reported peanuts kept weed free six weeks after planting had no yield loss when weeds emerged later [3].

To effectively control weeds, producers should develop integrated weed management practices that minimize environmental impacts without forfeiting profitability [9]. In commercial crop production, weed control is built on the use of herbicides [10]. Producers prefer to use a single-pass herbicide program to achieve season-long weed control, which reduces labor costs and time [11,12]. However, in the southeastern U.S., weed management relies heavily on preplant-incorporated (PPI)-, PRE-, and POST-applied herbicides [13]. Preplant-incorporated herbicides are assimilated into the soil 
prior to crop planting with mechanical incorporation [14]. Preemergence herbicides help to reduce yield loss from weed interference, allow time for crop establishment, and mitigate selection pressure to POST herbicides [15]. Postemergence herbicides are most effective when the application rate, application timing, and weed size at application are in compliance with the herbicide label [10].

The biology and competitive nature of weeds is critical for producers to understand when developing a weed management strategy. Producers that implement effective weed management strategies to control competitive weeds during the CPWC can potentially maximize crop yield [3,6,7]. Using multiple herbicide MOA can mitigate potential for crop yield loss and also reduce the potential for herbicide-resistant weeds to evolve [10].

To overcome yearly weed infestations, control of emerged seedlings and depletion of the soil seedbank is essential [16]. A soil seedbank is a reserve for viable seeds contained in the soil profile or soil surface in a given area [17]. Mature weeds will release seeds to the soil surface, many of which accumulate in the soil seedbank [16]. However, if weed seed production is prevented, weed soil seedbanks can be depleted [16]. In agricultural fields, the soil seedbank provides long-term persistence of weed seed that survive less than 1 year $[16,18,19]$. Weed seed in the soil seedbank may not germinate for several years due to dormancy traits [20]. Understanding seed-producing characteristics of weed species is critical for developing methods that mitigate the addition of weed seed to the soil [21].

Following a 4-yr fallow soil with intensive tillage, research reported that only $10 \%$ of the original velvetleaf [Abutilon throphrasti (Medik)] seed was still present in the soil [22]. Additional studies observed reported viable weed seeds were reduced in the soil by $95 \%$ across five sites in Nebraska following a 5-yr period of controlling weeds exclusively with herbicides [23]. Cultivated soils accommodate large amounts of weed seed that interfere with crop production, which forces long-term weed management strategies to focus on controlling emerged seedlings [16]. Weed seed replenishing the soil seedbank will increase weed populations in successive growing seasons; therefore, an increase in herbicide applications are needed to control those weed populations [24]. However, a system of weed management that completely controls aboveground weeds for a long period of time will not completely eliminate weeds from that area [25]. Therefore, weed management can only be successful when control methods are focused on manipulating the weed seedbank [26].

Controlling competitive weeds during early season peanut growth can reduce yield loss potential and mitigate weed seed additions to the seedbank. It is imperative for peanut producers to reduce weed seed dispersion to the soil seedbank. Allowing weeds to mature and produce seed may increase weed populations in successive growing seasons. Depletion of the soil seedbank by controlling weeds prior to producing seed can potentially reduce weed populations and reduce the amount of herbicide applications needed in successive growing seasons. Currently, herbicides are the most widely used weed control option for Mississippi peanut producers. Based on previous research and current field observations, multiple herbicide applications are needed to control weeds in Mississippi peanut. Increasing the number of herbicide applications may result in greater weed control and weed seed suppression. Therefore, a study was established at the Delta Research and Extension Center to evaluate various herbicide programs for controlling common weed species that interfere with peanut in Mississippi. The purpose of this study was to identify the best herbicide program for weed control and reducing weed seed production in a Mississippi peanut production system.

\section{Materials and Methods}

\subsection{Field Preparation and Study Design}

A field study was conducted at the Delta Research and Extension Center in Stoneville, MS, USA in 2017 and $2018\left(33^{\circ} 26^{\prime} 37.1^{\prime \prime} \mathrm{N}, 90^{\circ} 54^{\prime} 29.88^{\prime \prime} \mathrm{W}\right)$ to evaluate PRE and POST herbicide programs in Mississippi peanut. Soil series at each site was a Commerce silty clay loam (fine-silty, mixed, superactive, nonacid, thermic Fluvaquentic Endoaquepts) with a pH of 7.4 and an organic matter content of approximately $1.5 \%$. The 2017 and 2018 sites were fallow the previous year. Therefore, 
both sites had a variety of weed species that were allowed to mature and replenish the soil seedbank naturally. However, barnyardgrass, hemp sesbania, Palmer amaranth, pitted morningglory, and prickly sida seed were broadcasted both years to achieve uniform weed populations.

In both siteyears, fields were disked and beds were established. Paraquat (Gramoxone SL 2.0, herbicide, $841 \mathrm{~g}$ ai ha $^{-1}$, Syngenta Crop Protection, Greensboro, NC, USA) was applied immediately after planting to control existing weeds. In both siteyears, 'Georgia-06G' (University of Georgia-Coastal Plain Experiment Station, Tifton, GA, USA) was planted to a depth of $5 \mathrm{~cm}$ at a seeding rate of 258,300 seeds ha ${ }^{-1}$ using a small-plot planter (John Deere MaxEmerge Plus 1700, Deere and Company, Moline, IL, USA). Peanut were planted on 23 May and 5 May in 2017 and 2018, respectively. Plot size was $4 \times 6 \mathrm{~m}$ and consisted of four rows spaced $101.6 \mathrm{~cm}$ apart. All four rows received herbicide treatments, but only rows two and three were harvested.

The study was designed as a randomized complete block with four replications. Treatments were different combinations of herbicides which included acetochlor, clethodim, flumioxazin, lactofen, paraquat, and s-metolachlor (Table 1). Applications were made using a $\mathrm{CO}_{2}$-pressurized backpack sprayer equipped with turbo induction nozzles (Turbo TeeJet Induction 110015 nozzle, TeeJet Technologies, Springfield, IL, USA) set to deliver $140 \mathrm{~L} \mathrm{ha}^{-1}$ at $206 \mathrm{kPa}$. All treatments received a PRE application and a sequential application at 2 to 3 weeks after emergence (EPOST), 4 to 5 weeks after emergence (MPOST), or both EPOST and MPOST. A nontreated control was included.

\subsection{Data Collection}

Visible estimates of peanut injury and weed control were recorded $21 \mathrm{~d}$ after PRE (DA PRE), $7 \mathrm{~d}$ after EPOST (DA-EPOST), and 7 and $35 \mathrm{~d}$ after MPOST (DA-MPOST) on a scale of 0 to $100 \%$ where 0 indicated no visible effect and 100 indicated complete plant death. Prior to each application timing, weed density $\left(\mathrm{m}^{-2}\right)$ and height $(\mathrm{cm})$ were recorded for barnyardgrass, hemp sesbania, Palmer amaranth, pitted morningglory, and prickly sida (Table 2). 
Table 1. Herbicides used in this study.

\begin{tabular}{ccccc}
\hline WSSA Group & Common Name & Trade Name & Use Rate & Manufacturer \\
\hline 15 & acetochlor & Warrant & $1260 \mathrm{~g} \mathrm{ai} \mathrm{ha}^{-1}$ & Bayer CropScience LP, P.O. Box 12014, 2 T.W. Alexander Drive, Research Triangle Park, NC 27709 $^{-1}$ \\
1 & clethodim & Select Max & $59.5 \mathrm{~g} \mathrm{ai} \mathrm{ha}^{-1}$ & Valent U.S.A. Corporation, P.O. Box 8025 Walnut Creek, CA 94596 \\
14 & flumioxazin & Valor SX & $53.6 \mathrm{~g} \mathrm{ai} \mathrm{ha}^{-1}$ & Valent U.S.A. Corporation, P.O. Box 8025 Walnut Creek, CA 94596 \\
14 & lactofen & Cobra & $210 \mathrm{~g} \mathrm{ai} \mathrm{ha}^{-1}$ & Valent U.S.A. Corporation, P.O. Box 8025 Walnut Creek, CA 94596 \\
22 & paraquat & Gramoxone 2.0 SL & $210 \mathrm{~g} \mathrm{ai} \mathrm{ha}^{-1}$ & Syngenta Crop Protection, P.O. Box 18300 Greensboro, NC 27419 \\
15 & s-metolachlor & Dual Magnum & $1070 \mathrm{~g} \mathrm{ai} \mathrm{ha}^{-1}$ & Syngenta Crop Protection, P.O. Box 18300 Greensboro, NC 27419 \\
\hline
\end{tabular}

Table 2. Height and density of barnyardgrass, hemp sesbania, Palmer amaranth, pitted morningglory, and prickly sida in the Weed Control Study at Stoneville, MS, USA in 2017 and 2018.

\begin{tabular}{|c|c|c|c|c|c|c|c|c|c|c|c|}
\hline \multirow{2}{*}{ Year } & \multirow{2}{*}{ Timing } & \multicolumn{2}{|c|}{ Barnyardgrass } & \multicolumn{2}{|c|}{ Hemp sesbania } & \multicolumn{2}{|c|}{ Palmer amaranth } & \multicolumn{2}{|c|}{ Pitted morningglory } & \multicolumn{2}{|c|}{ Prickly sida } \\
\hline & & Height & Density & Height & Density & Height & Density & Height & Density & Height & Density \\
\hline & & $\mathrm{cm}$ & no. $\mathrm{m}^{-2}$ & $\mathrm{~cm}$ & no. $\mathrm{m}^{-2}$ & $\mathrm{~cm}$ & no. $\mathrm{m}^{-2}$ & $\mathrm{~cm}$ & no. $\mathrm{m}^{-2}$ & $\mathrm{~cm}$ & no. $\mathrm{m}^{-2}$ \\
\hline 2017 & EPOST $^{\mathrm{a}}$ & 8.4 & 35 & 8.4 & 3 & 6.9 & 29 & 7.62 & 6 & 4.6 & 6 \\
\hline 2017 & MPOST $^{a}$ & 13.2 & 4 & 13.5 & 3 & 18 & 3 & 9.7 & 0.5 & 10.7 & 1.5 \\
\hline 2018 & EPOST & 9.9 & 25 & 11.7 & 2 & 7.9 & 78 & 8.4 & 2 & 4.4 & 0.5 \\
\hline 2018 & MPOST & 7.9 & 4 & 18.5 & 3 & 15 & 13 & 11.2 & 2 & 5 & 0.5 \\
\hline
\end{tabular}

${ }^{\text {a }}$ Herbicide application timings to peanut were based on 2 to 3 weeks after emergence (EPOST) and 4 to 5 weeks after emergence (MPOST). 
Prior to peanut harvest, all weeds present in two 1- $\mathrm{m}^{2}$ quadrats in each plot were harvested by hand, placed in paper bags, dried for 8 weeks at $\geq 32{ }^{\circ} \mathrm{C}$, and then weighed. All weed species excluding Palmer amaranth were hand-threshed to remove seeds, and total seed weight for each species was recorded. Additionally, for each weed species except Palmer amaranth, five samples consisting of 100 seeds each were weighed to determine average seed weight for each species. The average seed weight for each species was then utilized to estimate the total seed $\mathrm{m}^{-2}$ in each plot. Total seed $\mathrm{m}^{-2}$ was calculated for barnyardgrass, hemp sesbania, pitted morningglory, and prickly sida with the following equation:

Total number of seeds $\mathrm{m}^{-2}=($ Total seed weight for sample $\times 100) /($ Weight of 100 seeds $)$

Palmer amaranth seed were removed by plant grinder (Thomas Model 4 Wiley Mill, Thomas Scientific, Swedesboro, NJ, USA) to determine total seed weight. Five 0.5-g samples of Palmer amaranth seed were counted to determine the average number of seed in each sample. The average seed number was then used to calculate the amount of seed produced $\mathrm{m}^{-2}$ in each plot. Average seed number for Palmer amaranth was calculated using the following equation:

Total number of Palmer amaranth seed $\mathrm{m}^{-2}=$ (Number of seed in $0.5 \mathrm{~g} \times$ Total weight of seed $\left.\mathrm{m}^{-2}\right) / 0.5$

To determine effectiveness of each herbicide program in reducing overall weed seed production, the following equation was used:

Percent weed seed reduction following each herbicide program $=$

[1 - (Total weed seed following each herbicide program/Total weed seed in nontreated control)]

Following weed sample collection, peanuts were unearthed by a digger. The peanut digger consisted of a blade underneath a conveyer belt to cut peanut taproot and overturn plants to be left on the soil surface. Peanut remained on the soil surface for 2 weeks before harvest. Rows two and three in each plot were unearthed and harvested to record peanut yield. In 2017, peanuts were harvested on November 20; however, inclement weather prevented peanut harvest in 2018.

\subsection{Statistical Analysis}

Square roots of visible estimates of peanut injury and weed control were arcsine transformed. The transformation did not improve homogeneity of variance; therefore, nontransformed data were used in analysis. Nontreated data were excluded when injury and weed control were analyzed. However, nontreated data were included in analysis for weed above-ground dry weight, weed seed production, and peanut yield. Data were pooled across siteyears and subjected to ANOVA using the PROC GLIMMIX procedure in SAS 9.4 (SAS Institute Inc., Cary, NC, USA) with year and replication (nested within year) as random effect parameters [27]. Least square means were calculated and mean separation $(p \leq 0.05)$ were produced in SAS, which converts mean separation output to letter groupings [28].

\section{Results and Discussion}

No peanut injury was observed following PRE applications. However, peanut injury was significant $(p<0.0001)$ at 7 DA-EPOST and 7 DA-MPOST applications. Peanut injury was observed when EPOST and MPOST treatments contained paraquat, lactofen, or lactofen plus clethodim. At 7 DA-EPOST, peanut injury was greatest from treatments containing paraquat EPOST (8 to $10 \%$ ). All remaining treatments causing peanut injury 7 DA-EPOST contained lactofen with injury from 2 to $6 \%$. Peanut injury 7 DA-MPOST was greatest following paraquat EPOST fb lactofen or lactofen plus clethodim MPOST with injury from 3 to $4 \%$. 


\subsection{Barnyardgrass Control}

Barnyardgrass control was significant at every evaluation. At 21 DA-PRE ( $p=0.0012)$, a difference among treatments was detected for barnyardgrass control (Table 3). S-metolachlor provided the greatest level of control (92 to 95\%) compared with flumioxazin and acetochlor (76 to 82\%). The greatest difference among PRE applications was flumioxazin and s-metolachlor which provided 82 and $95 \%$ barnyardgrass control, respectively. At 21 DA-PRE, s-metolachlor provided greater control of barnyardgrass than flumioxazin and acetochlor.

Barnyardgrass control was significant among treatments 7 DA-EPOST $(p<0.0001)$. Treatments providing similar control of barnyardgrass ranged from 91 to 100\% (Table 3). Additionally, comparable treatments that provided 99 to $100 \%$ control were different from flumioxazin PRE fb acetochlor EPOST $\mathrm{fb}$ lactofen plus clethodim MPOST and acetochlor PRE fb lactofen EPOST, which resulted in 60 and $85 \%$ control, respectively. Greatest difference in barnyardgrass control was observed following acetochlor PRE fb lactofen EPOST compared with flumioxazin PRE fb lactofen plus clethodim EPOST, s-metolachlor PRE fb lactofen plus clethodim EPOST, s-metolachlor PRE fb paraquat EPOST, and flumioxazin PRE fb lactofen plus clethodim EPOST fb acetochlor MPOST, which provided 85, 99, 100, 100, and 100\% control, respectively. At 7 DA-EPOST, differences in barnyardgrass control were observed for treatments containing lactofen or lactofen plus clethodim EPOST. At 7 DA-EPOST, acetochlor PRE fb lactofen EPOST compared with flumioxazin PRE fb lactofen plus clethodim EPOST, s-metolachlor PRE fb lactofen plus clethodim EPOST, and flumioxazin PRE fb lactofen plus clethodim EPOST fb acetochlor MPOST resulted in 85, 99, 100, and 100\% control, respectively.

Differences in barnyardgrass control were detected 7 DA-MPOST $(p<0.0001)$. Treatments providing similar control of barnyardgrass 7 DA-MPOST ranged from 85 to $96 \%$ (Table 3). Treatments providing 91 to $96 \%$ control were different from acetochlor PRE fb lactofen MPOST, flumioxazin PRE $\mathrm{fb}$ paraquat EPOST, flumioxazin PRE fb paraquat EPOST fb acetochlor MPOST, and flumioxazin PRE $\mathrm{fb}$ acetochlor EPOST fb lactofen plus clethodim MPOST, which resulted in 68, 69, 70, and 77\% control of barnyardgrass, respectively. The greatest difference among treatments for barnyardgrass control were observed following flumioxazin PRE fb acetochlor EPOST fb lactofen plus clethodim MPOST and flumioxazin PRE fb paraquat EPOST fb lactofen plus clethodim MPOST, which provided 77 and $96 \%$ control, respectively. The greatest difference in barnyardgrass control 35 DA-MPOST was observed following acetochlor PRE fb s-metolachlor plus paraquat EPOST fb lactofen MPOST and flumioxazin PRE fb paraquat EPOST fb lactofen plus clethodim MPOST which provided 69 and 93\% control, respectively.

\subsection{Hemp Sesbania Control}

Hemp sesbania control was significant $(p<0.0001$ to $p=0.0102) 21$ DA-PRE, 7 DA-EPOST, and 7 and 35 DA-MPOST. At 21 DA-PRE, all treatments provided $\geq 90 \%$ control of hemp sesbania (Table 4). At 7 DA-EPOST, all treatments provided comparable control of hemp sesbania (97 to $100 \%$ ) except $s$-metolachlor PRE fb lactofen plus clethodim MPOST, which provided $86 \%$ control. For evaluations 7 DA-MPOST, comparable control among treatments ranged from 95 to $100 \%$. However, treatments providing $100 \%$ control 7 DA-EPOST were different from s-metolachlor PRE fb paraquat EPOST and flumioxazin PRE fb paraquat EPOST fb acetochlor MPOST which provided 89 and $94 \%$ control of hemp sesbania, respectively. Hemp sesbania control 35 DA-MPOST $(p<0.0001)$ was different among treatments. All treatments with comparable control of hemp sesbania 35 DA-MPOST ranged from 89 to $100 \%$. Treatments providing 100\% control of hemp sesbania were different from s-metolachlor PRE fb paraquat EPOST and s-metolachlor PRE fb lactofen plus clethodim MPOST, which provided 60 and $84 \%$ control. 
Table 3. Control of barnyardgrass 21 DA-PRE, 7 DA-EPOST ${ }^{\mathrm{b}}$, and 7 and 35 DA-MPOST ${ }^{\mathrm{b}}$ treatments in Weed Control Study at Stoneville, MS averaged over 2017 and $2018^{\mathrm{a}}$.

\begin{tabular}{|c|c|c|c|c|}
\hline Herbicide Programs & 21 DA-PRE & 7 DA-EPOST & 7 DA-MPOST & 35 DA-MPOST \\
\hline flumioxazin PRE fb paraquat ${ }^{c}$ EPOST & $79 \mathrm{~b}$ & $94 \mathrm{ab}$ & $69 c$ & 50 e \\
\hline S-metolachlor PRE fb paraquat EPOST & $92 \mathrm{a}$ & $100 \mathrm{a}$ & $88 \mathrm{ab}$ & $83 \mathrm{ab}$ \\
\hline flumioxazin PRE fb $S$-metolachlor + paraquat EPOST & $78 \mathrm{~b}$ & $96 \mathrm{ab}$ & $85 \mathrm{ab}$ & 63 cde \\
\hline flumioxazin PRE fb lactofen + clethodim ${ }^{\mathrm{d}}$ EPOST & $76 \mathrm{~b}$ & 99 a & $88 \mathrm{ab}$ & $75 \mathrm{abc}$ \\
\hline flumioxazin PRE fb lactofen + clethodim MPOST & $82 \mathrm{~b}$ & $91 \mathrm{ab}$ & $88 \mathrm{ab}$ & $88 \mathrm{ab}$ \\
\hline S-metolachlor PRE fb lactofen + clethodim EPOST & $94 \mathrm{a}$ & $100 \mathrm{a}$ & $95 \mathrm{a}$ & $90 \mathrm{a}$ \\
\hline S-metolachlor PRE fb lactofen + clethodim MPOST & $95 \mathrm{a}$ & $94 \mathrm{ab}$ & $88 \mathrm{ab}$ & $86 \mathrm{ab}$ \\
\hline acetochlor PRE fb lactofen ${ }^{\mathrm{d}}$ EPOST & $79 \mathrm{~b}$ & $85 \mathrm{~b}$ & $68 c$ & $49 \mathrm{e}$ \\
\hline flumioxazin PRE fb paraquat EPOST fb lactofen + clethodim MPOST & $81 \mathrm{~b}$ & $96 \mathrm{ab}$ & $96 \mathrm{a}$ & 93 a \\
\hline acetochlor PRE fb S-metolachlor + paraquat EPOST fb lactofen MPOST & $78 \mathrm{~b}$ & $95 \mathrm{ab}$ & $85 \mathrm{ab}$ & $69 \mathrm{bcd}$ \\
\hline flumioxazin PRE fb paraquat EPOST fb acetochlor MPOST & $81 \mathrm{~b}$ & $96 \mathrm{ab}$ & $70 \mathrm{c}$ & 50 de \\
\hline flumioxazin PRE fb lactofen + clethodim EPOST fb acetochlor MPOST & $80 \mathrm{~b}$ & $100 \mathrm{a}$ & $91 \mathrm{a}$ & $88 \mathrm{a}$ \\
\hline flumioxazin PRE fb acetochlor EPOST fb lactofen + clethodim MPOST & $81 \mathrm{~b}$ & $60 c$ & $77 \mathrm{bc}$ & $75 \mathrm{abc}$ \\
\hline
\end{tabular}

a All data pooled over two siteyears. Means within a column followed by the same letter are not different at $p \leq 0.05 .{ }^{\mathrm{b}}$ EPOST treatments were applied 2 to 3 weeks after emergence. MPOST treatments were applied 4 to 5 weeks after emergence. ${ }^{c}$ All paraquat treatments included non-ionic surfactant (NIS) at $0.25 \% v / v$. ${ }^{\mathrm{d}}$ All lactofen and lactofen plus clethodim treatments included crop oil concentrate (COC) at $1 \% v / v$. 
Table 4. Control of hemp sesbania 21 DA-PRE, 7 DA-EPOST ${ }^{b}$, and 7 and 35 DA-MPOST ${ }^{b}$ treatments in Weed Control Study at Stoneville, MS, averaged over 2017 and $2018^{\mathrm{a}}$.

\begin{tabular}{|c|c|c|c|c|}
\hline \multirow[b]{3}{*}{ flumioxazin PRE fb paraquat ${ }^{c}$ EPOST } & 21 DA-PRE & 7 DA-EPOST & 7 DA-MPOST & 35 DA-MPOST \\
\hline & \multicolumn{4}{|c|}{ 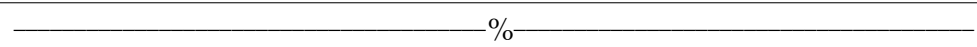 } \\
\hline & $98 \mathrm{a}$ & 99 a & $99 \mathrm{ab}$ & $95 \mathrm{ab}$ \\
\hline S-metolachlor PRE fb paraquat EPOST & $94 \mathrm{bc}$ & 98 a & $89 \mathrm{c}$ & $60 c$ \\
\hline flumioxazin PRE fb S-metolachlor + paraquat EPOST & $97 \mathrm{ab}$ & $98 \mathrm{a}$ & $96 \mathrm{ab}$ & $95 \mathrm{ab}$ \\
\hline flumioxazin PRE fb lactofen + clethodim ${ }^{\mathrm{d}}$ EPOST & 99 a & $100 \mathrm{a}$ & $99 \mathrm{ab}$ & $98 \mathrm{ab}$ \\
\hline flumioxazin PRE fb lactofen + clethodim MPOST & $99 \mathrm{a}$ & $97 \mathrm{a}$ & $100 \mathrm{a}$ & $100 \mathrm{a}$ \\
\hline S-metolachlor PRE fb lactofen + clethodim EPOST & $94 \mathrm{bc}$ & $100 \mathrm{a}$ & $98 \mathrm{ab}$ & $92 \mathrm{ab}$ \\
\hline S-metolachlor PRE fb lactofen + clethodim MPOST & $93 \mathrm{bcd}$ & $86 \mathrm{~b}$ & $97 \mathrm{ab}$ & $84 \mathrm{~b}$ \\
\hline acetochlor PRE fb lactofen ${ }^{\mathrm{d}}$ EPOST & $91 \mathrm{~cd}$ & $100 \mathrm{a}$ & $98 \mathrm{ab}$ & $91 \mathrm{ab}$ \\
\hline flumioxazin PRE fb paraquat EPOST fb lactofen + clethodim MPOST & $100 \mathrm{a}$ & $100 \mathrm{a}$ & $100 \mathrm{a}$ & $100 \mathrm{a}$ \\
\hline acetochlor PRE fb S-metolachlor + paraquat EPOST fb lactofen MPOST & $90 \mathrm{~d}$ & $97 \mathrm{a}$ & $95 \mathrm{ab}$ & $89 \mathrm{ab}$ \\
\hline flumioxazin PRE fb paraquat EPOST fb acetochlor MPOST & $97 \mathrm{ab}$ & 98 a & $94 \mathrm{bc}$ & $89 a b$ \\
\hline flumioxazin PRE fb lactofen + clethodim EPOST fb acetochlor MPOST & $97 \mathrm{ab}$ & $99 \mathrm{a}$ & $98 \mathrm{ab}$ & $96 \mathrm{ab}$ \\
\hline flumioxazin PRE fb acetochlor EPOST fb lactofen + clethodim MPOST & $99 \mathrm{a}$ & $98 \mathrm{a}$ & $100 \mathrm{a}$ & $100 \mathrm{a}$ \\
\hline
\end{tabular}

${ }^{\text {a }}$ All data pooled over two siteyears. Means within a column followed by the same letter are not different at $p \leq 0.05 .{ }^{b}$ EPOST treatments were applied 2 to 3 weeks after emergence. MPOST treatments were applied 4 to 5 weeks after emergence. ${ }^{c}$ All paraquat treatments included NIS at $0.25 \% v / v$. d All lactofen and lactofen plus clethodim treatments included COC at $1 \%$ v $/ v$. 


\subsection{Palmer Amaranth Control}

Palmer amaranth control was significant following treatments 21 DA-PRE $(p=0.0044), 7$ DA-EPOST $(p<0.0001), 7$ DA-MPOST $(p=0.0004)$, and 35 DA-MPOST $(p<0.0001)$. At 21 DA-PRE, flumioxazin provided 94 to $96 \%$ control of Palmer amaranth (Table 5). Acetochlor and s-metolachlor provided similar control of Palmer amaranth ranging from 85 to $91 \%$. All treatments 7 DA-EPOST provided comparable control (93 to 100\%) except s-metolachlor PRE fb lactofen plus clethodim MPOST, which provided 73\% control of Palmer amaranth. At 7 DA-MPOST, treatments providing 95 to $98 \%$ control were different from treatments providing 83 to $89 \%$ control of Palmer amaranth. The greatest difference among treatments was detected following acetochlor PRE fb lactofen EPOST and flumioxazin PRE fb paraquat EPOST fb lactofen plus clethodim MPOST, which provided 89 and 98\% control of Palmer amaranth, respectively. At 35 DA-MPOST, treatments with comparable Palmer amaranth control ranged from 88 to $96 \%$. Treatments providing 94 to $96 \%$ were different from s-metolachlor PRE fb lactofen plus clethodim MPOST, s-metolachlor PRE fb lactofen plus clethodim EPOST, and s-metolachlor PRE fb paraquat EPOST which provided 74, 78, and 83\%, respectively. The greatest difference in control of Palmer amaranth was following s-metolachlor PRE fb paraquat EPOST compared with flumioxazin PRE $\mathrm{fb}$ paraquat EPOST fb lactofen plus clethodim MPOST and flumioxazin PRE fb lactofen plus clethodim EPOST fb acetochlor MPOST, which provided 83, 96, and 96\% control.

\subsection{Pitted Morningglory Control}

Pitted morningglory control was significant 21 DA-PRE $(p<0.0001), 7$ DA-EPOST $(p<0.0001)$, and $7(p=0.0002)$ and $35(p<0.0001)$ DA-MPOST. At 21 DA-PRE, flumioxazin provided 96 to $99 \%$ control of pitted morningglory (Table 6). Acetochlor and s-metolachlor provided comparable control of pitted morningglory which ranged from 86 to $89 \%$. At 7 DA-EPOST, similar treatments provided 97 to $100 \%$ which were different from s-metolachlor PRE fb lactofen plus clethodim MPOST which provided $87 \%$ control. Treatments providing 99 to $100 \%$ control 7 DA-EPOST were different from $s$-metolachlor PRE fb lactofen plus clethodim MPOST and flumioxazin PRE fb lactofen plus clethodim MPOST which provided 87 and $94 \%$ control. At 7 DA-MPOST, all treatments provided $\geq 91 \%$ control of pitted morningglory. Treatments providing 98 to $100 \%$ control of pitted morningglory 7 DA-MPOST were different from $s$-metolachlor PRE fb lactofen plus clethodim MPOST and acetochlor PRE fb lactofen EPOST which provided 91 and $92 \%$ control. At 35 DA-MPOST, comparable control of pitted morningglory among treatments ranged from 92 to $99 \%$ control. Treatments providing 92 to $100 \%$ control of pitted morningglory were different from s-metolachlor PRE fb lactofen plus clethodim MPOST and s-metolachlor PRE fb paraquat EPOST, which provided 72 and $78 \%$ control, respectively.

\subsection{Prickly Sida Control}

Prickly sida control was significant 21 DA-PRE, 7 DA-EPOST, and 35 DA-MPOST. At 21 DA-PRE $(p<0.0001)$, all treatments provided $\geq 93 \%$ control of prickly sida (Table 7). All treatments 7 DA-EPOST $(p=0.0192)$ provided $\geq 98 \%$ control of prickly sida. At 35 DA-MPOST $(p=0.0039)$, all treatments provided $\geq 94 \%$ control of prickly sida. 
Table 5. Control of Palmer amaranth 21 DA-PRE, 7 DA-EPOST ${ }^{b}$, and 7 and 35 DA-MPOST ${ }^{b}$ treatments in Weed Control Study at Stoneville, MS, averaged over 2017 and $2018^{\mathrm{a}}$.

\begin{tabular}{|c|c|c|c|c|}
\hline Herbicide Programs & 21 DA-PRE & 7 DA-EPOST & 7 DA-MPOST & 35 DA-MPOST \\
\hline \multirow[b]{2}{*}{ flumioxazin PRE fb paraquat ${ }^{c}$ EPOST } & \multicolumn{3}{|c|}{$-\%$} & \\
\hline & $94 \mathrm{ab}$ & $100 \mathrm{a}$ & $95 \mathrm{ab}$ & $94 \mathrm{a}$ \\
\hline$S$-metolachlor PRE fb paraquat EPOST & $91 \mathrm{a}-\mathrm{d}$ & 99 a & $86 \mathrm{~cd}$ & $83 c$ \\
\hline flumioxazin PRE fb $S$-metolachlor + paraquat EPOST & $94 \mathrm{ab}$ & $100 \mathrm{a}$ & $96 a b$ & $92 \mathrm{ab}$ \\
\hline flumioxazin PRE fb lactofen + clethodim ${ }^{\mathrm{d}}$ EPOST & $94 \mathrm{ab}$ & $100 \mathrm{a}$ & $97 \mathrm{ab}$ & $91 \mathrm{ab}$ \\
\hline flumioxazin PRE fb lactofen + clethodim MPOST & $94 \mathrm{ab}$ & $93 \mathrm{a}$ & $94 \mathrm{abc}$ & 94 a \\
\hline S-metolachlor PRE fb lactofen + clethodim EPOST & $88 \mathrm{~cd}$ & $96 a$ & $84 \mathrm{~d}$ & $78 \mathrm{c}$ \\
\hline S-metolachlor PRE fb lactofen + clethodim MPOST & $87 \mathrm{~d}$ & $73 \mathrm{~b}$ & $83 \mathrm{~d}$ & $74 \mathrm{c}$ \\
\hline acetochlor PRE fb lactofen ${ }^{\mathrm{d}}$ EPOST & $88 \mathrm{~cd}$ & 96 a & $89 \mathrm{bcd}$ & $88 \mathrm{~b}$ \\
\hline flumioxazin PRE fb paraquat EPOST fb lactofen + clethodim MPOST & $94 \mathrm{ab}$ & $100 \mathrm{a}$ & 98 a & $95 \mathrm{a}$ \\
\hline acetochlor PRE fb S-metolachlor + paraquat EPOST fb lactofen MPOST & $85 \mathrm{~d}$ & 99 a & $96 \mathrm{ab}$ & $93 \mathrm{ab}$ \\
\hline flumioxazin PRE fb paraquat EPOST fb acetochlor MPOST & $96 \mathrm{a}$ & $100 \mathrm{a}$ & 98 a & $96 \mathrm{a}$ \\
\hline flumioxazin PRE fb lactofen + clethodim EPOST fb acetochlor MPOST & $94 \mathrm{ab}$ & $100 \mathrm{a}$ & 98 a & 96 a \\
\hline flumioxazin PRE fb acetochlor EPOST fb lactofen + clethodim MPOST & $95 \mathrm{a}$ & $95 \mathrm{a}$ & $91 \mathrm{abcd}$ & $91 \mathrm{ab}$ \\
\hline
\end{tabular}

${ }^{\text {a }}$ All data pooled over two siteyears. Means within a column followed by the same letter are not different at $p \leq 0.05 .{ }^{\mathrm{b}}$ EPOST treatments were applied 2 to 3 weeks after emergence. MPOST treatments were applied 4 to 5 weeks after emergence. ${ }^{c}$ All paraquat treatments included NIS at $0.25 \% v / v$. ${ }^{\mathrm{d}}$ All lactofen and lactofen plus clethodim treatments included COC at $1 \% v / v$. 
Table 6. Control of pitted morningglory 21 DA-PRE, 7 DA-EPOST, and 7 and 35 DA-MPOST ${ }^{\mathrm{b}}$ treatments in Weed Control Study at Stoneville, MS, averaged over 2017 and $2018^{\mathrm{a}}$.

\begin{tabular}{|c|c|c|c|c|}
\hline \multirow[b]{3}{*}{ flumioxazin PRE fb paraquat ${ }^{c}$ EPOST } & 21 DA-PRE & 7 DA-EPOST & 7 DA-MPOST & 35 DA-MPOST \\
\hline & \multicolumn{4}{|c|}{$\ldots \%$} \\
\hline & $96 \mathrm{ab}$ & $100 \mathrm{a}$ & $100 \mathrm{a}$ & $98 \mathrm{ab}$ \\
\hline S-metolachlor PRE fb paraquat EPOST & $88 \mathrm{~d}$ & $97 \mathrm{ab}$ & $94 \mathrm{bcd}$ & $78 \mathrm{~cd}$ \\
\hline flumioxazin PRE fb S-metolachlor + paraquat EPOST & $96 \mathrm{ab}$ & $100 \mathrm{a}$ & $98 \mathrm{ab}$ & $96 \mathrm{ab}$ \\
\hline flumioxazin PRE fb lactofen + clethodim ${ }^{\mathrm{d}}$ EPOST & $96 \mathrm{ab}$ & $100 \mathrm{a}$ & $96 \mathrm{abc}$ & $92 \mathrm{ab}$ \\
\hline flumioxazin PRE fb lactofen + clethodim MPOST & $96 \mathrm{ab}$ & $94 \mathrm{~b}$ & $96 \mathrm{abc}$ & $97 \mathrm{ab}$ \\
\hline S-metolachlor PRE fb lactofen + clethodim EPOST & $89 \mathrm{~cd}$ & $97 \mathrm{ab}$ & $95 \mathrm{abcd}$ & $85 \mathrm{bc}$ \\
\hline S-metolachlor PRE fb lactofen + clethodim MPOST & $93 \mathrm{bc}$ & $87 \mathrm{c}$ & $91 \mathrm{~d}$ & $72 \mathrm{~d}$ \\
\hline acetochlor PRE fb lactofen ${ }^{\mathrm{d}}$ EPOST & $89 \mathrm{~cd}$ & $98 \mathrm{ab}$ & $92 \mathrm{~cd}$ & $94 \mathrm{ab}$ \\
\hline flumioxazin PRE fb paraquat EPOST fb lactofen + clethodim MPOST & 99 a & $100 \mathrm{a}$ & $100 \mathrm{a}$ & $100 \mathrm{a}$ \\
\hline acetochlor PRE fb S-metolachlor + paraquat EPOST fb lactofen MPOST & $86 \mathrm{~d}$ & 99 a & 99 a & $95 \mathrm{ab}$ \\
\hline flumioxazin PRE fb paraquat EPOST fb acetochlor MPOST & $98 \mathrm{a}$ & $100 \mathrm{a}$ & $100 \mathrm{a}$ & $98 \mathrm{ab}$ \\
\hline flumioxazin PRE fb lactofen + clethodim EPOST fb acetochlor MPOST & $97 \mathrm{a}$ & $100 \mathrm{a}$ & $100 \mathrm{a}$ & $98 \mathrm{ab}$ \\
\hline flumioxazin PRE fb acetochlor EPOST fb lactofen + clethodim MPOST & 99 a & $98 \mathrm{ab}$ & 99 a & 99 a \\
\hline
\end{tabular}

${ }^{\text {a }}$ All data pooled over two siteyears. Means within a column followed by the same letter are not different at $p \leq 0.05 .{ }^{\mathrm{b}}$ EPOST treatments were applied 2 to 3 weeks after emergence. MPOST treatments were applied 4 to 5 weeks after emergence. ${ }^{c}$ All paraquat treatments included NIS at $0.25 \% v / v$. ${ }^{\mathrm{d}}$ All lactofen and lactofen plus clethodim treatments included COC at $1 \% v / v$. 
Table 7. Control of prickly sida 21 DA-PRE, 7 DA-EPOST ${ }^{\mathrm{b}}$, and 7 and 35 DA-MPOST ${ }^{\mathrm{b}}$ treatments in Weed Control Study at Stoneville, MS averaged over 2017 and $2018^{\mathrm{a}}$.

\begin{tabular}{|c|c|c|c|c|}
\hline Herbicide Programs & 21 DA-PRE & 7 DA-EPOST & 7 DA-MPOST & 35 DA-MPOST \\
\hline flumioxazin PRE fb paraquat ${ }^{c}$ EPOST & $100 \mathrm{a}$ & $100 \mathrm{a}$ & $100 \mathrm{a}$ & $100 \mathrm{a}$ \\
\hline S-metolachlor PRE fb paraquat EPOST & $98 \mathrm{ab}$ & $100 \mathrm{a}$ & $100 \mathrm{a}$ & $94 \mathrm{c}$ \\
\hline flumioxazin PRE fb $S$-metolachlor + paraquat EPOST & $99 \mathrm{ab}$ & $100 \mathrm{a}$ & $100 \mathrm{a}$ & $100 \mathrm{a}$ \\
\hline flumioxazin PRE fb lactofen + clethodim ${ }^{\mathrm{d}}$ EPOST & $100 \mathrm{a}$ & $100 \mathrm{a}$ & $100 \mathrm{a}$ & $100 \mathrm{a}$ \\
\hline flumioxazin PRE fb lactofen + clethodim MPOST & $100 \mathrm{a}$ & $100 \mathrm{a}$ & $100 \mathrm{a}$ & $100 \mathrm{a}$ \\
\hline$S$-metolachlor PRE fb lactofen + clethodim EPOST & $98 \mathrm{ab}$ & $100 \mathrm{a}$ & $100 \mathrm{a}$ & $96 \mathrm{bc}$ \\
\hline S-metolachlor PRE fb lactofen + clethodim MPOST & $100 \mathrm{a}$ & $98 \mathrm{~b}$ & $100 \mathrm{a}$ & $94 \mathrm{c}$ \\
\hline acetochlor PRE fb lactofen EPOST ${ }^{d}$ & 98 a & $99 \mathrm{ab}$ & $100 \mathrm{a}$ & $100 \mathrm{a}$ \\
\hline flumioxazin PRE fb paraquat EPOST fb lactofen + clethodim MPOST & $100 \mathrm{a}$ & $100 \mathrm{a}$ & $100 \mathrm{a}$ & $100 \mathrm{a}$ \\
\hline acetochlor PRE fb S-metolachlor + paraquat EPOST fb lactofen MPOST & $93 \mathrm{c}$ & $100 \mathrm{a}$ & $100 \mathrm{a}$ & $100 \mathrm{a}$ \\
\hline flumioxazin PRE fb paraquat EPOST fb acetochlor MPOST & $100 \mathrm{a}$ & $100 \mathrm{a}$ & $100 \mathrm{a}$ & $100 \mathrm{a}$ \\
\hline flumioxazin PRE fb lactofen + clethodim EPOST fb acetochlor MPOST & $99 \mathrm{ab}$ & $100 \mathrm{a}$ & $100 \mathrm{a}$ & $100 \mathrm{a}$ \\
\hline flumioxazin PRE fb acetochlor EPOST fb lactofen + clethodim MPOST & $100 \mathrm{a}$ & $100 \mathrm{a}$ & $100 \mathrm{a}$ & $99 a b$ \\
\hline
\end{tabular}

a All data pooled over two siteyears. Means within a column followed by the same letter are not different at $p \leq 0.05 .{ }^{\mathrm{b}}$ EPOST treatments were applied 2 to 3 weeks after emergence. MPOST treatments were applied four to five weeks after emergence. ${ }^{c}$ All paraquat treatments included NIS at $0.25 \% \mathrm{v} / \mathrm{v}$. ${ }^{\mathrm{d}}$ All lactofen and lactofen plus clethodim treatments included $\mathrm{COC}$ at $1 \% v / v$. 


\subsection{Peanut Yield}

Peanut were not harvested in 2018 due to inclement weather. However, there was a peanut yield difference among treatments in $2017(p<0.0001)$. Peanut yield among treatments ranging from 2680 to $3130 \mathrm{~kg} \mathrm{ha}^{-1}$ were not different, but were different from treatments resulting in peanut yield of 982 to $2087 \mathrm{~kg} \mathrm{ha}^{-1}$ (data not shown). Weed interference resulted in an $88 \%$ reduction in peanut yield when the nontreated control (368 $\left.\mathrm{kg} \mathrm{ha}^{-1}\right)$ was compared to flumioxazin PRE fb lactofen plus clethodim MPOST (3126 kg ha $\left.{ }^{-1}\right)$.

\subsection{Pitted Morningglory and Hemp Sesbania: Above-Ground Dry Weight and Seed Production}

Prior to peanut harvest, weed above ground dry weight (AGDW) was significant for hemp sesbania $(p<0.0001)$ and pitted morningglory $(p=0.0009)$. Differences in seed production were detected for barnyardgrass $(p=0.0002)$, hemp sesbania $(p<0.0001)$, Palmer amaranth $(p=0.0443)$, and pitted morningglory $(p=0.031)$. Hemp sesbania and pitted morningglory were the only two species with differences for both AGDW and seed production (Table 8). Hemp sesbania AGDW was $153 \mathrm{~g}$ and produced 1942 seeds $\mathrm{m}^{-2}$ for the nontreated control. Flumioxazin PRE fb paraquat EPOST $\mathrm{fb}$ acetochlor MPOST provided the least reduction of AGDW, which resulted in $86.12 \mathrm{~g} \mathrm{~m}^{-2}$. However, $S$-metolachlor PRE fb paraquat EPOST was the worst treatment in terms of seed production which resulted in 1046 seeds $\mathrm{m}^{-2}$ deposited to the soil seedbank. Ten treatments provided comparable reduction in hemp sesbania seed production ranging from 0 to 207 seeds $\mathrm{m}^{-2}$.

For pitted morningglory, all treatments reduced AGDW from 0 to $23 \mathrm{~g} \mathrm{~m}^{-2}$ (Table 8). Pitted morningglory following s-metolachlor PRE fb lactofen plus clethodim MPOST resulted in AGDW of $23 \mathrm{~g} \mathrm{~m}^{-2}$ which was different from treatments resulting in 0 to $8 \mathrm{~g} \mathrm{~m}^{-2}$ of AGDW. S-metolachlor PRE $\mathrm{fb}$ paraquat EPOST resulted in comparable reduction of pitted morningglory AGDW $\left(15 \mathrm{~g} \mathrm{~m}^{-2}\right)$ as $s$-metolachlor PRE fb lactofen plus clethodim MPOST. All treatments resulted in pitted morningglory seed production ranging from 0 to 138 seeds $\mathrm{m}^{-2}$. Acetochlor PRE fb lactofen EPOST (138 seeds $\mathrm{m}^{-2}$ ) was different from all treatments that resulted in pitted morningglory seed production ranging from 0 to 55 seeds $\mathrm{m}^{-2}$. The only comparable treatment to acetochlor PRE fb lactofen EPOST was flumioxazin $\mathrm{PRE} \mathrm{fb}$ paraquat EPOST $\mathrm{fb}$ acetochlor MPOST, which resulted in pitted morningglory producing 63 seeds $\mathrm{m}^{-2}$. 
Table 8. Above-ground dry weight of hemp sesbania and pitted morningglory $\mathrm{m}^{-2}$, as well as seed production for barnyardgrass, hemp sesbania, Palmer amaranth, and pitted morningglory $\mathrm{m}^{-2}$ following PRE, EPOST ${ }^{\mathrm{b}}$, and MPOST ${ }^{\mathrm{b}}$ herbicide programs prior to peanut harvest in Stoneville, MS, averaged over 2017 and $2018^{\mathrm{a}}$.

\begin{tabular}{|c|c|c|c|c|c|c|}
\hline \multirow[t]{2}{*}{ Herbicide Programs } & \multicolumn{2}{|c|}{ Above-Ground Dry Weight } & \multicolumn{4}{|c|}{ Seed Number $\mathbf{m}^{-2}$} \\
\hline & $\begin{array}{l}\text { Hemp } \\
\text { sesbania }\end{array}$ & $\begin{array}{l}\text { Pitted } \\
\text { morningglory }\end{array}$ & Barnyardgrass & $\begin{array}{l}\text { Hemp } \\
\text { sesbania }\end{array}$ & $\begin{array}{l}\text { Palmer } \\
\text { amaranth }\end{array}$ & $\begin{array}{l}\text { Pitted } \\
\text { morningglory }\end{array}$ \\
\hline flumioxazin PRE fb paraquat ${ }^{c}$ EPOST & $11.2 \mathrm{c}$ & $g \longdiv { 3 . 9 c }$ & 13,696 a & $79 c$ & $6350 \mathrm{bc}$ & $0 \mathrm{~b}$ \\
\hline S-metolachlor PRE fb paraquat EPOST & $31.9 \mathrm{bc}$ & $15 \mathrm{ab}$ & $5038 \mathrm{c}-\mathrm{g}$ & $1046 \mathrm{~b}$ & $12,294 \mathrm{bc}$ & $36 \mathrm{~b}$ \\
\hline flumioxazin PRE fb $S$-metolachlor + paraquat EPOST & $36.4 \mathrm{bc}$ & $5.5 \mathrm{bc}$ & $10,225 \mathrm{abc}$ & $132 \mathrm{c}$ & $14,411 \mathrm{bc}$ & $2 \mathrm{~b}$ \\
\hline flumioxazin PRE fb lactofen + clethodim $^{\mathrm{d}}$ EPOST & $10.9 \mathrm{c}$ & $5.9 \mathrm{bc}$ & 8624 a-e & $135 c$ & $27,138 \mathrm{bc}$ & $23 \mathrm{~b}$ \\
\hline flumioxazin PRE fb lactofen + clethodim MPOST & $10 \mathrm{c}$ & $0 \mathrm{c}$ & $2239 \mathrm{~g}$ & $0 \mathrm{c}$ & $6673 \mathrm{bc}$ & $0 \mathrm{~b}$ \\
\hline S-metolachlor PRE fb lactofen + clethodim EPOST & $8.8 \mathrm{c}$ & $7.2 \mathrm{bc}$ & $4448 \mathrm{~d}-\mathrm{g}$ & $207 c$ & $16,764 \mathrm{bc}$ & $23 \mathrm{~b}$ \\
\hline S-metolachlor PRE fb lactofen + clethodim MPOST & $52.4 \mathrm{~b}$ & $22.8 \mathrm{a}$ & $2375 \mathrm{fg}$ & $433 \mathrm{bc}$ & $30,420 \mathrm{ab}$ & 138 a \\
\hline acetochlor PRE fb lactofen ${ }^{\mathrm{d}}$ EPOST & $13.3 \mathrm{c}$ & $2.4 \mathrm{c}$ & $7593 \mathrm{~b}-\mathrm{f}$ & $74 \mathrm{c}$ & $11,876 \mathrm{bc}$ & $1 \mathrm{~b}$ \\
\hline flumioxazin PRE fb paraquat EPOST fb lactofen + clethodim MPOST & $4.3 \mathrm{c}$ & $3.8 \mathrm{c}$ & $6293 \mathrm{~b}-\mathrm{g}$ & $0 \mathrm{c}$ & $6184 \mathrm{bc}$ & $7 \mathrm{~b}$ \\
\hline $\begin{array}{c}\text { acetochlor PRE fb S-metolachlor + paraquat EPOST fb lactofen MPOST } \\
\text { Table } 8 \text { (continued) }\end{array}$ & $12.9 \mathrm{c}$ & $1.1 \mathrm{c}$ & $8939 \mathrm{a}-\mathrm{d}$ & $163 c$ & $14,770 \mathrm{bc}$ & $0 \mathrm{~b}$ \\
\hline flumioxazin PRE fb paraquat EPOST fb acetochlor MPOST & $86.2 \mathrm{~b}$ & $8 \mathrm{bc}$ & $10,245 \mathrm{abc}$ & $743 \mathrm{bc}$ & $11,793 \mathrm{bc}$ & $63 \mathrm{ab}$ \\
\hline flumioxazin PRE fb lactofen + clethodim EPOST fb acetochlor MPOST & $6.9 \mathrm{c}$ & $3.6 \mathrm{c}$ & $4208 \mathrm{~d}-\mathrm{g}$ & $79 \mathrm{c}$ & $538 \mathrm{c}$ & $55 \mathrm{~b}$ \\
\hline flumioxazin PRE fb acetochlor EPOST fb lactofen + clethodim MPOST & $0 \mathrm{c}$ & $0 \mathrm{c}$ & 3469 efg & $0 \mathrm{c}$ & $8243 \mathrm{bc}$ & $0 \mathrm{~b}$ \\
\hline Nontreated Control & $153.1 \mathrm{a}$ & $7.8 \mathrm{bc}$ & $11,064 \mathrm{ab}$ & $1942 \mathrm{a}$ & 58,196 a & $43 \mathrm{~b}$ \\
\hline
\end{tabular}

${ }^{a}$ All data pooled over two siteyears. Means within a column followed by the same letter are not different at $p \leq 0.05 .{ }^{b}$ EPOST treatments were applied 2 to 3 weeks after emergence. MPOST

treatments were applied 4 to 5 weeks after emergence. ${ }^{c}$ All paraquat treatments included NIS at $0.25 \% v / v$. ${ }^{d}$ All lactofen and lactofen plus clethodim treatments included COC at $1 \% v / v$. 


\subsection{Barnyardgrass Seed Production}

Barnyardgrass AGDW was not affected by the treatments imposed in this study (data now shown). However, treatments were different in terms of reducing seed production (Table 8). All treatments resulted in barnyardgrass seed production from 2239 to 13,696 seeds $\mathrm{m}^{-2}$. Barnyardgrass seed production following flumioxazin PRE fb lactofen plus clethodim MPOST resulted in the greatest suppression of seed production (2239 seeds $\mathrm{m}^{-2}$ ). Flumioxazin PRE fb paraquat EPOST resulted in the greatest level of barnyardgrass seed production at 13,696 seeds $\mathrm{m}^{-2}$.

\subsection{Palmer Amaranth Seed Production}

Palmer amaranth AGDW was not affected by the treatments imposed in this study (data not shown). Palmer amaranth produced 58,196 seeds $\mathrm{m}^{-2}$ in the nontreated control (Table 8 ). All treatments provided comparable reduction of Palmer amaranth seed production ranging from 538 to 30,420 seeds $\mathrm{m}^{-2}$. Flumioxazin PRE fb lactofen plus clethodim EPOST fb acetochlor MPOST, which resulted in 538 Palmer amaranth seeds $\mathrm{m}^{-2}$, was the only treatment different from $s$-metolachlor PRE fb lactofen plus clethodim MPOST, which resulted in 30,420 seeds $\mathrm{m}^{-2}$. Flumioxazin PRE fb lactofen plus clethodim EPOST $\mathrm{fb}$ acetochlor MPOST reduced Palmer amaranth seed $\geq 99 \%$ compared with the nontreated control.

\subsection{Prickly Sida Seed Production}

Prickly sida AGDW and seed production were not affected by treatments in this study (data not shown). However, several treatments effectively reduced or eliminated seed production of prickly sida. In the nontreated control, prickly sida produced 4621 seeds $\mathrm{m}^{-2}$. All treatments reduced prickly sida seed production from 0 to 3263 seeds $\mathrm{m}^{-2}$.

\subsection{Summary of Results}

The current research demonstrated that herbicide programs provide different levels of control, AGDW, and seed production of barnyardgrass, hemp sesbania, Palmer amaranth, pitted morningglory, and prickly sida in Mississippi peanut.

Final evaluations recorded 35 DA-MPOST for barnyardgrass detected a difference in treatments that contained paraquat applied EPOST (Table 3). Flumioxazin PRE fb paraquat EPOST and flumioxazin PRE fb paraquat plus s-metolachlor EPOST resulted in 50 and $63 \%$ control, respectively. However, $s$-metolachlor PRE fb paraquat EPOST provided 83\% control of barnyardgrass 35 DA-MPOST. $S$-metolachlor PRE provided greater control of barnyardgrass than flumioxazin PRE; therefore, when s-metolachlor PRE fb paraquat EPOST was applied, greater control of barnyardgrass was achieved 35 DA-MPOST than treatments containing flumioxazin PRE fb paraquat or paraquat plus $s$-metolachlor EPOST. Comparable treatments to s-metolachlor PRE fb paraquat EPOST were observed following s-metolachlor PRE fb lactofen plus clethodim MPOST, flumioxazin PRE fb lactofen plus clethodim MPOST, s-metolachlor PRE fb lactofen plus clethodim EPOST, and flumioxazin PRE fb paraquat EPOST fb lactofen plus clethodim MPOST which provided 86, 88, 90, and 93\% control 35 DA-MPOST, respectively. The lowest level of control among comparable treatments were acetochlor PRE fb lactofen EPOST, flumioxazin PRE fb paraquat EPOST, flumioxazin PRE fb paraquat EPOST fb acetochlor MPOST, and flumioxazin PRE fb paraquat plus s-metolachlor EPOST, which provided 49, 50,50 , and $63 \%$ control of barnyardgrass 35 DA-MPOST.

For Palmer amaranth control 35 DA-MPOST, nine treatments provided comparable control ranging from 91 to $96 \%$ control (Table 5). At 35 DA-MPOST, s-metolachlor PRE fb lactofen plus clethodim MPOST, s-metolachlor PRE fb lactofen plus clethodim EPOST, and s-metolachlor PRE fb paraquat EPOST provided only 74, 78, and 83\% control of Palmer amaranth. All treatments containing flumioxazin and acetochlor applied PRE controlled Palmer amaranth greater than treatments containing $s$-metolachlor PRE. 
At 35 DA-MPOST, 11 treatments provided comparable control of hemp sesbania ranging from 89 to $100 \%$ control (Table 4). At 35 DA-MPOST, s-metolachlor PRE fb paraquat EPOST provided $60 \%$ control of hemp sesbania, which was the lowest level of control. Pitted morningglory control 35 DA-MPOST ranged from 72 to 100\% following all treatments (Table 6). Ten treatments provided comparable control of pitted morningglory ranging from 92 to $100 \%$ at 35 DA-MPOST. All treatments provided 94 to $100 \%$ control of prickly sida 35 DA-MPOST.

The treatments in this two-year study resulted in differences for control, AGDW, and weed seed production among all weed species. Previous research reported that weeds developed herbicide resistance by responding to overreliance of a single pest control method $[10,29,30]$. Additionally, research stated that multiple modes of action applied simultaneously or sequentially can reduce the potential for weeds to develop herbicide resistance [10]. Therefore, weeds exposed to treatments such as flumioxazin PRE fb lactofen plus clethodim MPOST may have the potential to develop resistance due to flumioxazin and lactofen being WSSA group 14 herbicides (Table 1). However, treatments such as flumioxazin PRE fb paraquat EPOST fb lactofen plus clethodim or flumioxazin PRE fb lactofen plus clethodim EPOST $\mathrm{fb}$ acetochlor MPOST may help to reduce the risk for weeds to develop herbicide resistance due to an additional herbicide with a different mode of action included in both treatments.

Several treatments in this study resulted in some weed species producing zero seed (Table 8 ). Even if treatments in this study provided weed control $\geq 95 \%$, weeds that matured and produced seed may have replenished the soil seedbank. Several treatments reduced weed seed production, but did not completely eliminate the possibility of increasing weed populations in successive growing seasons [25]. If weed seed replenishes the soil seedbank, an increase in herbicide applications may be needed in sequential growing seasons to control them [24]. To effectively control weeds and deplete the soil seedbank, weed management strategies must implement methods for reducing the addition of weed seed to the soil seedbank [21]. Therefore, weed management strategies cannot be focused on aboveground vegetation alone, but must implement practices, such as tillage, to reduce the soil seedbank [22,26].

\section{Conclusions}

Among all weed species, the most consistent control was provided by flumioxazin PRE fb lactofen plus clethodim MPOST, flumioxazin PRE fb lactofen plus clethodim EPOST fb acetochlor MPOST, and flumioxazin PRE fb paraquat EPOST fb lactofen plus clethodim MPOST, which provided 88, 88, and 93\% control 35 DA-MPOST. All treatments reduced hemp sesbania AGDW as compared to the nontreated control. However, pitted morningglory following s-metolachlor PRE fb lactofen plus clethodim MPOST had greater AGDW than the nontreated control and was different from all other treatments except s-metolachlor PRE fb paraquat EPOST. Treatments with PRE and MPOST or PRE, EPOST, and MPOST applications resulted in 82 to $93 \%$ reduction in weed seed production. Therefore, weed control programs must include a PRE and MPOST application to reduce weed seed production greater than PRE fb EPOST programs.

Author Contributions: Conceptualization and methodology of the projects, T.B.; data collection was conducted by J.W.S. and T.B.; formal data analysis was conducted by J.W.S., J.A.B., B.R.G., and J.G.; writing-review and editing, J.W.S., J.A.B., T.B., J.G., and B.R.G. All authors have read and agreed to the published version of the manuscript.

Funding: Mississippi Peanut Promotion Board.

Acknowledgments: This publication is a contribution of the Delta Research and Extension Center and Mississippi Agricultural and Forestry Experiment Station. This material is based upon work that is supported by the Mississippi Peanut Promotion Board, Hatch project under accession number 230080.

Conflicts of Interest: The authors declare no conflict of interest. 


\section{Nomenclature}

acetochlor: clethodim, flumioxazin, lactofen, lactofen plus clethodim, paraquat, S-metolachlor; barnyardgrass, Echinochloa crus-galli L. Beauv. ECHCG; hemp sesbania, Sesbania herbacea (P. Mill.) McVaugh SEBEX; Palmer amaranth, Amaranthus palmeri S. Watts AMAPA; pitted morningglory, ipomoea lacunosa L. IPOLA; prickly sida, Sida spinosa L. SIDSP; peanut, Arachis hypogea L.

\section{References}

1. Bajwa, A.A.; Mahajan, G.; Chauhan, B.S. Nonconventional weed management strategies for modern agriculture. Weed Sci. 2015, 63, 723-747. [CrossRef]

2. Everman, W.J.; Burke, I.C.; Clewis, S.B.; Thomas, W.E.; Wilcut, J.W. Critical period of grass vs. broadleaf weed interference in peanut. Weed Technol. 2008, 22, 68-73. [CrossRef]

3. Hill, L.V.; Santelmann, P.W. Competitive effects of annual weeds on Spanish peanuts. Weed Sci. 1969, 17, 1-2. [CrossRef]

4. Hauser, E.W.; Buchanan, G.A.; Ethredge, W.J. Competition of Florida beggarweed and sicklepod with peanuts I. effects of periods of weed-free maintenance or weed competition. Weed Sci. 1975, 23, 368-372. [CrossRef]

5. Buchanan, G.A.; Hauser, E.W.; Ethredge, W.J.; Cecil, S.R. Competition of Florida Beggarweed and Sicklepod with peanuts II. Effects of cultivation, weeds, and SADH. Weed Sci. 1976, 24, 29-39. [CrossRef]

6. Knezevic, S.Z.; Evans, S.P.; Blankenship, E.E.; Van Acker, R.C.; Lindquist, J.L. Critical period for weed control: The concept and data analysis. Weed Sci. 2002, 50, 773-786. [CrossRef]

7. Kasasian, L.; Seeyave, J. Critical periods of weed competition. Proc. Acad. Natl. Sci. USA 1969, 15, $208-212$. [CrossRef]

8. Zimdahl, R.L. Weed-Crop Competition: A Review; International Plant Protection Center, Oregon State University: Corvallis, OR, USA, 1980; 195p.

9. Buhler, D.D.; Gunsolus, J.L.; Ralston, D.F. Integrated weed management techniques to reduce herbicide inputs in soybean. Agron. J. 1992, 84, 973-978. [CrossRef]

10. Norsworthy, J.K.; Ward, S.M.; Shaw, D.R.; Llewellyn, R.S.; Nichols, R.L.; Webster, T.M.; Bradley, K.W.; Frisvold, G.; Powles, S.B.; Burgos, N.R.; et al. Reducing the risks of herbicide resistance: Best management practices and recommendations. Weed Sci. 2012, 60, 31-62. [CrossRef]

11. Jordan, D.L. Influence of adjuvants on the antagonism of graminicides by broadleaf herbicides. Weed Technol. 1995, 9, 741-747. [CrossRef]

12. Nurse, R.E.; Hamill, A.S.; Swanton, C.J.; Tardif, F.J.; Deen, W.; Sikkema, P.H. Is the application of a residual herbicide required prior to glyphosate application in no-till glyphosate-tolerant soybean? Crop Prot. 2007, 26, 484-489. [CrossRef]

13. Wilcut, J.; York, A.; Grichar, W.; Wehtje, G. The biology and management of weeds in peanut (Arachis hypogaea). In Advances in Peanut Science; Pattee., H.E., Stalker, H.E., Eds.; American Peanut Research and Education Society Inc.: Stillwater, OK, USA, 1995; Chapter 6; pp. 221-223.

14. McCloskey, W.; Brown, L. Preplant and Preemergence Dinitroaniline Herbicides in Cotton. 2013. Available online: https://cals.arizona.edu/crop/cotton/files/DNA_Herbicides.pdf (accessed on 29 June 2020).

15. Jhala, A.; Kruger, G. Why Residual Herbicides are Important Tools for Battling Hard-to-Control Weeds. 2015. Available online: https:/cropwatch.unl.edu/why-residual-herbicides-are-important-tools-battling-hardcontrol-weeds (accessed on 19 February 2019).

16. Smith, C.W.; Betran, J.; Runge, E.C.A. (Eds.) Corn: Origin, History, Technology, and Production; John Wiley \& Sons, Inc.: Hoboken, NJ, USA, 2004; pp. 719-721.

17. Roberts, H.A. Seed banks in the soil. Adv. Appl. Biol. 1981, 6, 1-55.

18. Cardina, J.; Herms, C.; Doohan, D.J. Crop rotation and tillage system effects on weed seedbanks. Weed Sci. 2002, 50, 448-460. [CrossRef]

19. Forcella, F.; Wilson, R.G.; Renner, K.A.; Dekker, J.; Harvey, R.G.; Alm, D.A. Weed seedbanks of the U.S. corn belt: Magnitude, variation, emergence, and application. Weed Sci. 1992, 40, 636-644. [CrossRef]

20. Baskin, J.M.; Baskin, C.C. Role of temperature in regulating timing of germination in soil seed reserves of Thlaspi arvense L. Weed Res. 1984, 24, 341-349. [CrossRef] 
21. Schwartz, L.M.; Norsworthy, J.K.; Young, B.G.; Bradley, K.W.; Kruger, G.R.; Davis, V.M.; Steckel, L.E.; Walsh, M.J. Tall waterhemp (Amaranthus tuberculatus) and Palmer amaranth (Amaranthus palmeri) seed production and retention at soybean maturity. Weed Technol. 2016, 30, 284-290. [CrossRef]

22. Lueschen, W.E.; Andersen, R.N. Longevity of velvetleaf (Abutilon theophrasti) seeds in soil under agricultural practices. Weed Sci. 1980, 28, 341-346. [CrossRef]

23. Burnside, O.C.; Moomaw, R.S.; Roeth, F.W.; Wicks, G.A. Weed seed demise in soil in weed-free corn (Zea mays) production across Nebraska. Weed Sci. 1986, 34, 248-251. [CrossRef]

24. Norsworthy, J.K.; Oliver, L.R. Hemp sesbania interference in drill-seeded glyphosate-resistant soybean. Weed Sci. 2002, 50, 34-41. [CrossRef]

25. Chancellor, R.L. The manipulation of weed behaviour for control purposes. Philos. Trans. R. Soc. B 1981, 295, 103-110.

26. Kremer, R.J. Management of weed seed banks with microorganisms. Ecol. Appl. 1993, 3, 42-52. [CrossRef]

27. Blouin, D.C.; Webster, E.P.; Bond, J.A. On the analysis of combined experiments. Weed Technol. 2011, 25, 165-169. [CrossRef]

28. Saxton, A.M. A macro for converting mean separation output to letter groupings in PROC MIXED. In Proceedings of the 23rd SAS Users Group International, Nashville, TN, USA, 22-25 March 1998; pp. 1243-1246.

29. Harrington, J.; Byrne, P.F.; Peairs, F.B.; Nissen, S.J.; Westra, P.; Ellsworth, P.C.; Fournier, A.; Mallory-Smith, C.A.; Zemetra, R.S.; Henry, W.B. Perceived consequences of herbicide-tolerant and insect-resistant crops on integrated pest management strategies in the western United States: Results of an online survey. AgBioForum 2009, 12, 412-421.

30. Vencill, W.K.; Nichols, R.L.; Webster, T.M.; Soteres, J.K.; Mallory-Smith, C.; Burgos, N.R.; Johnson, W.G.; McClelland, M.R. Herbicide resistance: Toward an understanding of resistance development and the impact of herbicide-resistant crops. Weed Sci. 2012, 60, 2-30. [CrossRef]

(C) 2020 by the authors. Licensee MDPI, Basel, Switzerland. This article is an open access article distributed under the terms and conditions of the Creative Commons Attribution (CC BY) license (http://creativecommons.org/licenses/by/4.0/). 